Revista de Psicología de la PUCP. Vol. XIII. No 1. 1995

\title{
CONCEPTOS BASICOS Y RENDIMIENTO ESCOLAR EN NIÑAS DE PRIMER GRADO DE PRIMARIA DE CLASE SOCIOECONOMICA BAJA
}

\author{
María Isabel La Rosa Cormack ${ }^{1}$
}

La finalidad de este trabajo fue resolver la interrogante acerca de la relación entre el conocimiento de conceptos básicos de espacio, tiempo, cantidad y número, y el rendimiento escolar, a parrir de un diseño de tipo correlacional. Para ello se tomó una muestra compuesta por treinta niñas de seis años de edad que cursaban el primer grado de primaria, pertenecientes a la clase socioeconómica baja. Como instrumentos se utilizaron el Test Boehm de Conceptos Básicos y una Prueba de Pedagógica que evaluaría el desempeño tanto en lenguaje como en matemáticas. Los resultados mostraron una correlación significativa entre las variables del estudio siendo mayor, la correlación entre conceptos y lenguaje, que entre conceptos y matemáticas. Palabras Claves: Prueba de Conceptos Básicos de Boehm, rendimiento escolar, niñas, nivel socioeconómico bajo.

Basic Concepts and school performance in girls at first grade and low SES

The finality of this work was to solve the question about the relationship between knowledge of basic concepts such as: space, time quantity and number, and school performance. The sample was conformed by 30 low school first grade students aged 6 years belonging to low SES. The Boehm Test of Basic Concepts and a Pedagogical Test were administred to asses performance in language and mathematics. Significant correlation was founded between the study variables being higher the relation about concepts and language than concepts and mathematics.

Key words: Test Boehm of Basic Concepts, school performance, girls, low SES.

1. Licenciada en Psicología. Profesora Auxiliar del Departamento de Humanidades Pontificia Universidad Católica del Perú, Coordinadora de Psicología ante la Facultad de LL y CC HH. Dirección: Sección Psicología, Apartado 1761, Lima 100-Perú. Internet mlarosa@pucp.edu.pe. 

En el niño, los conceptos se van formando a través de una selección de ejemplos apropiados y el rechazo de aquéllos que resultan inapropiados. Es así que, cuando un concepto es formulado en una palabra, ello implica un acto de generalización. Esta capacidad de generalizar, que tienen los seres humanos, a partir de hechos o situaciones particulares, es lo que permite el aprendizaje de conceptos. Este aprendizaje permite tratar a los eventos que tienen propiedades comunes como si fueran miembros de una clase. Cuando además, lo podemos aplicar a un gran número de casos y en forma correcta, entonces podemos decir que se ha adquirido el concepto.

No obstante, lograr el desarrollo y definición de algunos conceptos es probable que requiera un tiempo prolongado. Así mismo, un concepto particular se puede agudizar o extender con experiencia adicional, entrenamiento avanzado o conocimiento nuevo. Por ejemplo, para Vigotsky (1992), el proceso de formación de un concepto, es más que la suma de determinados enlaces asociativos formados por la memoria, es más que un simple hábito mental; es un acto de pensamiento complejo y genuino, que puede verificarse cuando el desarrollo de éste ha alcanzado el nivel requerido. Para Oerter (1975) en este proceso, el niño inicialmente, sólo será capaz de tener en cuenta los ejemplos positivos, para luego incluir sistemáticamente la información negativa.

Ausubel (1976) indica que hay dos tipos principales de adquisición de conceptos: (1) La formación de conceptos, que es el proceso que tiene que ver con la abstracción de los rasgos comunes y esenciales, donde interviene el análisis discriminativo, la abstracción, diferenciación y generalización. (2) La asimilación conceptual, en la que se aprenden nuevos significados conceptuales y se relacionan con los ya existentes en el repertorio el sujeto. 
La formación de conceptos ha sido estudiada desde diferentes perspectivas: asociacionista, mediacionista y cognoscitiva. Las teorías asociacionistas explican la conducta conceptual en base al principio de condicionamiento. Según esto, la formación de conceptos constituye un proceso mediante el cual, el individuo establece nexos o relaciones entre cada ejemplo que encuentra o se le presenta, ya sea positivo o negativo y la forma como lo ubica en categorías. Bourne y Restle (1975) indican, que el principal parámetro estructural de esta teoría es la tasa de formación de asociaciones y la adaptación, que se considera una función de la proporción de elementos pertinentes en la población total de elementos. Por otra parte, el aprendizaje dentro de este enfoque considera que la conducta conceptual implica principalmente procesos de generalización y discriminación. Este aprendizaje de conceptos es considerado como una combinación de la discriminación entre clases de eventos y la generalización dentro de clases de eventos, para ello se varía no sólo lo que es pertinente al concepto, sino también lo que no es pertinente.

Las teorías mediacionistas enfatizan en la asociación entre una respuesta común a una clase de estímulos y la respuesta manifiesta. Los procesos mediadores son eventos simbólicos, que sirven para representar aspectos particulares de eventos de estimulación externa, por lo cual constituyen el eslabón que funciona entre el estímulo y la respuesta.

Por otra parte, tenemos la propuesta de Bruner (1966) que se enmarca en la perspectiva cognoscitiva, en la que los conceptos pueden entenderse como reglas de clasificación, que permiten agrupar objetos teniendo en cuenta determinadas características. Inicialmente, en el niño la representación se produce a través de la acción con su propio cuerpo, de su actividad motora con los objetos y los acontecimientos de los que dispone. A la representación a través de la acción, le sigue la representación icónica, entendida como la capacidad de producir imágenes precisas de su entorno. Un tercer nivel de complejidad es el que corresponde a la representación simbólica que resulta decisiva para la conceptualización propiamente dicha, en la que el lenguaje juega un rol fundamental. Por su parte, Ellis (1980) señala que el aprendizaje de muchos conceptos implica progresar de un estado difuso y grueso, a una condición altamente refinada, en la que se pueden hacer distinciones muy claras; así mismo nos permite 
sintetizar los puntos de vista antes propuestos, al señalar que el aprendizaje de conceptos como tema de la psicología del aprendizaje, está a medio camino, entre los procesos más simples de discriminación de estímulos, aprendizaje de respuestas y formación de asociaciones y los procesos más complejos de pensamiento, razonamiento y solución de problemas.

Aún cuando el problema de la relación entre pensamiento y lenguaje es controvertido, para Vigotsky (1964) el lenguaje no puede ser "descubierto" sin el pensamiento y considera decisivo su papel en la formación de los procesos mentales, sobre todo cuando señala que el método básico para analizar el desarrollo de las funciones psicológicas elevadas, es la reorganización de los procesos mentales, que tienen lugar bajo la influencia del lenguaje. Coincidentemente, Ausubel (1981) resalta el rol del lenguaje como facilitador en la adquisición de conceptos, lo que en su momento también ha sido destacado por Boehm $(1971,1986)$ considerando además, que éstos son fundamentales para el desempeńo escolar, ya que constituyen un soporte indispensable para la asimilación de nuevos procedimientos.

Otro factor de notable importancia en este proceso es la estimulación ambiental, la cual está directamente relacionada con el grupo familiar, ya que al interior de la familia ocurre gran parte de la estimulación del lenguaje, por lo tanto también influye en la adquisición del lenguaje y su desarrollo. A mayor carencia de estimulación ambiental se aprecia un nivel más bajo de desarrollo el lenguaje, que indudablemente va a repercutir en el proceso de formación de conceptos en el nińo. Bernstein (1967) señaló los contrastes entre el "lenguaje restringido" característico de los individuos de clase baja y los "mensajes complejos" propios de la clase media. Cuando se observa a las madres de clase socioeconómica baja, se aprecia que emplean un lenguaje sencillo carente de complicación gramatical, cuya característica son los mensajes cortos, fáciles, de entender; mientras que por el contrario, cuando se observa a las madres de clase socioeconómica media, éstas utilizan el lenguaje para socializar al nińo, para comunicarle sus emociones y sentimientos o para trasmitirle normas o pautas de disciplina.

Mussen, Conger y Kagan (1982) también observaron los efectos de la privación cultural y su influencia en el desarrollo del lenguaje puntualizando que una inadecuada estimulación unida a una interacción verbal 
pobre conllevará retrasos en el desarrollo cognoscitivo, en el área intelectual $y$ en el rendimiento escolar.

Gran parte del enfoque que se le da a la enseñanza, apunta al desarrollo de conceptos, pues son necesarios para el aprendizaje de principios, solución de problemas y actividades simbólicas como el pensamiento. Sin embargo, cuando hay carencia de estimulación ambiental, esto repercute en el desempeño escolar del niño, provocando en muchos casos la repitencia escolar, pues carece de los conceptos básicos que le permitirían tener un aprendizaje adecuado. La habilidad para manejar los conceptos, tal como existen en la realidad y tratar de ocuparse de ellos en una forma mucho más flexible y cambiante, es un objetivo común de la enseñanza en la escuela.

Boehm (1980) sostiene que el alumno que empieza el aprendizaje escolar con desventaja, es decir que carece de ciertos conceptos básicos a diferencia del grupo, suele mantener esta desventaja e incluso tiende a acentuarse, en la medida que los mismos pueden formar parte del nuevo aprendizaje, dificultando así su comprensión y asimilación. Todo esto es de suma importancia, sobre todo si se tienen en cuenta sus implicancias en el rendimiento escolar, tanto inicial como futuro.

Narváez (1987) en un estudio comparativo entre niños y niñas usando la Prueba de conceptos básicos de Boehm encontró que la variable socioeconómica incide significativamente en el desempeño de los sujetos evaluados, ya que a más alto nivel socioeconómico mejores o más altos puntajes en la prueba y a más bajo nivel socioeconómico más bajos puntajes, medias y desviación estándar. Thorne y Narváez (1987) señalan que a partir de la adaptación de la Prueba de Conceptos Básicos de Boehm podemos contar con un instrumento válido y confiable para nuestro medio, teniendo en cuenta como Marín (1986) la "individualidad etnocultural de nuestros pueblos". Para determinar el nivel de confiabilidad del instrumento, a partir del análisis de consistencia interna se utilizó la fórmula de Kuder-Richardson 20 y se obtuvo una correlación $\mathrm{r}=.8494$ entre los reactivos, los que permite indicar que se trata de un instrumento homogéneo. El estudio acerca de la validez de los items permitió reubicar 14 de los 50 reactivos, de tal forma que se mantuviera el criterio establecido por Boehm (1971) que señala que los 25 primeros son más fáciles que los 102 
25 últimos. La validez del instrumento fue analizada en términos de validez de contenido y aplicando el criterio de jueces se encontró un $86 \%$ de coincidencia. Se elaboraron Baremos para Lima y Callao.

De otro lado, la evaluación de los conceptos explicativos y ordenadores de causalidad, espacio, tiempo y número tienen en la infancia importancia trascendental en las tareas cotidianas de la escuela, ya que únicamente su existencia posibilita la realización de muchas funciones.

En este contexto, la importancia del presente trabajo residió en la posibilidad de verificar la relación entre el conocimiento de conceptos básicos y el rendimiento escolar, lo que puede formularse de la siguiente manera: ¿ Influye el conocimiento de conceptos básicos en el rendimiento escolar de las niñas de primer grado?. A partir de esta pregunta las hipótesis planteadas fueron las siguientes:

1. Ha: "Existe relación significativas entre los puntajes obtenidos en la Prueba de Conceptos Básicos de Boehm y los puntajes obtenidos en la Prueba Pedagógica".

2. Ha: "Existe relación significativa entre los puntajes obtenidos en la Prueba de Conceptos Básicos de Boehm y los puntajes obtenidos en la Prueba Pedagógica de lenguaje".

3. Ha: "Existe relación significativa entre los puntajes obtenidos en la Prueba de Conceptos Básicos de Boehm y los puntajes obtenidos en la Prueba Pedagógica de matemáticas".

\section{Metodología}

\section{Muestra}

Estuvo constituida por 30 niñas de seis años de edad, que cursaban el primer grado de primaria pertenecientes a la clase socioeconómica baja. Esta muestra se caracterizó por ser no probabilística e intencionada (Arnau, 1986). 


\section{Instrumentos}

- Prueba de Conceptos Básicos de Boehm (PCBB) forma A, que consta de dos cuadernillos El Cuadernillo 1 tiene 25 reactivos de dificultad creciente y el cuadernillo 2 consta también de 25 reactivos de dificultad creciente, siendo el Cuadernillo 2 más difícil que el 1. Los reactivos de la prueba agrupan los conceptos en cuatro categorías: relaciones espaciales, nociones de cantidad y número, nociones temporales, otros (conceptos no incluidos en las categorías antes mencionadas). Se utilizó la versión adaptadas y validada para nuestro medio por Narváez (1987).

- Prueba Pedagógica de Lenguaje y Matemáticas (PP) elaborada en base a los objetivos de la Estructura Curricular de Educación Primaria y la Guía Metodológica correspondiente. También se tuvo en cuenta la entrevista realizada con la profesora del aula para conocer hasta que objetivo había desarrollo en el Programa. La prueba consta de 20 reactivos, divididos en dos áreas: lenguaje (10 reactivos) y matemáticas (10 reactivos). Los reactivos de lenguaje evaluaron escritura, dictado y copiado. Los reactivos de matemáticas estaban referidos a conceptos numéricos, correspondencia términos a término, mayor, menor $\mathrm{e}$ igual, así como agrupar elementos de acuerdo a una consigna.

\section{Procedimiento}

Una vez determinada la muestra del estudio se procedió a la aplicación de los instrumentos de evaluación. La administración de la PCBB se hizo en forma individual y el tiempo aproximado que empleó cada niña para resolverla fue de 15 minutos, para los dos cuadernillos. Se dieron las siguientes instrucciones: "Voy a mostrarte un cuadernillo con dibujos. Vas a mirarlos y me vas a señalar lo que te pregunte". La instrucción fue similar para ambos cuadernillos. La administración de la prueba pedagógica se hizo en forma colectiva, dado que se consideró que las niñas ya estaban familiarizadas con este tipo de evaluaciones que se realizan en la escuela. Se les dieron las siguientes instrucciones: "En esta hoja que les he dado van a escribir su nombre y sus apellidos"... "ahora van a escribir lo que les voy a dictar"... "Copien lo que está escrito en la pizarra" (lenguaje); :"Vamos 104 
a resolver los ejercicios de esta prueba" (matemáticas). Se dio una instrucción específica para cada reactivo.

\section{Resultados}

Para el tratamiento estadístico de los datos (puntajes obtenidos por los sujetos en ambas pruebas), se utilizó la medida de correlación lineal de Pearson (r). En un primer análisis se estableció la correlación entre los puntajes de la PCBB y los puntajes globales obtenidos en la PP (lenguaje y matemáticas). Esta medida de correlación también se aplicó para determinar el sentido en que se daba dicha correlación. La correlación obtenida fue positiva y de, 77 entre las variables, es decir una correlación significativa (Ver cuadro 1). De otro lado, para verificar la primera Hipótesis se aplicó la prueba $t$ de student, con $\mathrm{p}<0.01$ y 28 grados de libertad. La t obtenida fue 6.36 lo cual implica una relación significativa entre los puntajes en la PCBB y los puntajes en la PP.

Un segundo análisis se hizo en relación a la segunda hipótesis, es decir determinar la relación entre los puntajes obtenidos en la PCBB y los puntajes obtenidos en la PP-lenguaje. Fue aplicada la medida de correlación de Person obteniéndose una correlación de .76, que indica una buena correlación entre las variables conceptos y lenguaje. Así mismo, se aplicó la prueba $t$ con un $\mathrm{p}<0.01$ y 28 grados de libertad. La obtenida fue de 6.18 , por lo tanto, existe una relación significativa entre estas variables. (Ver Cuadro 2).

Un tercer análisis correspondió a la tercera hipótesis para determinar la relación entre los puntajes obtenidos en la PCBB y los puntajes obtenidos en la PP-matemáticas. Con la aplicación de la medida de correlación de Pearson se obtuvo un coeficiente de .66, que si bien es adecuado, es más bajo que el establecido con respecto a la variable lenguaje. En este caso también se aplicó la prueba t para la verificación de la respectiva hipótesis con un $p<0.01$ y 28 grados de libertad. La t obtenida fue de 4.65 , lo que implica que existe una relación significativa entre las variables evaluadas. (Ver Cuadro 3). 
Cuadro 1: Puntajes en la PCBB (X) y en la Prueba Pedagógica (Y)

\begin{tabular}{|lrrrrr|}
\hline $\mathrm{n}$ & $\mathrm{X}$ & $\mathrm{Y}$ & $\mathrm{Xi}^{2}$ & $\mathrm{Yi}^{2}$ & $\mathrm{XiY}$ \\
\hline 1 & 32 & 15 & 1024 & 225 & 480 \\
2 & 33 & 14 & 1089 & 196 & 462 \\
3 & 38 & 14 & 1444 & 196 & 532 \\
4 & 36 & 12 & 1296 & 144 & 432 \\
5 & 38 & 12 & 1444 & 144 & 456 \\
6 & 26 & 4 & 676 & 16 & 104 \\
7 & 25 & 7 & 625 & 49 & 175 \\
8 & 22 & 4 & 484 & 16 & 88 \\
9 & 31 & 14 & 961 & 196 & 434 \\
10 & 30 & 11 & 900 & 121 & 330 \\
11 & 29 & 3 & 841 & 9 & 87 \\
12 & 33 & 6 & 529 & 36 & 138 \\
13 & 28 & 13 & 784 & 169 & 363 \\
14 & 26 & 4 & 676 & 16 & 104 \\
15 & 42 & 18 & 1764 & 324 & 756 \\
16 & 23 & 12 & 529 & 144 & 276 \\
17 & 41 & 18 & 1681 & 324 & 738 \\
18 & 26 & 5 & 676 & 25 & 130 \\
19 & 31 & 11 & 961 & 121 & 341 \\
20 & 20 & 8 & 400 & 64 & 160 \\
21 & 33 & 13 & 1089 & 169 & 429 \\
22 & 37 & 10 & 1369 & 100 & 370 \\
23 & 23 & 4 & 529 & 16 & 92 \\
24 & 40 & 14 & 1600 & 196 & 560 \\
25 & 28 & 13 & 784 & 169 & 364 \\
26 & 32 & 14 & 1024 & 196 & 448 \\
27 & 16 & 0 & 256 & 0 & 0 \\
28 & 33 & 11 & 1029 & 121 & 363 \\
29 & 32 & 12 & 1024 & 144 & 384 \\
30 & 29 & 5 & 841 & 25 & 145 \\
\hline & 901 & 301 & 28389 & 3671 & 9742 \\
\hline
\end{tabular}

$\mathrm{p}<0.01$ 
Cuadro 2: Puntajes en la PCBB (X) y en la PP de Lenguaje ( $\mathrm{Y}$ )

\begin{tabular}{|c|c|c|c|c|c|}
\hline $\mathrm{n}$ & $X$ & $Y$ & $\mathrm{Xi}^{2}$ & $\mathrm{Yi}^{2}$ & XiYi \\
\hline 1 & 32 & 8 & 1024 & 64 & 256 \\
\hline 2 & 33 & 6 & 1089 & 36 & 198 \\
\hline 3 & 38 & 7 & 1444 & 49 & 266 \\
\hline 4 & 36 & 7 & 1296 & 49 & 266 \\
\hline 5 & 38 & 5 & 1444 & 25 & 180 \\
\hline 6 & 26 & 1 & 676 & 1 & 26 \\
\hline 7 & 25 & 2 & 625 & 8 & 50 \\
\hline 8 & 22 & 2 & 484 & 4 & 44 \\
\hline 9 & 31 & 5 & 961 & 25 & 155 \\
\hline 10 & 30 & 5 & 900 & 16 & 120 \\
\hline 11 & 29 & 1 & 841 & 1 & 29 \\
\hline 12 & 33 & 1 & 529 & 1 & 23 \\
\hline 13 & 28 & 5 & 784 & 25 & 140 \\
\hline 14 & 26 & 1 & 676 & 1 & 26 \\
\hline 15 & 42 & 9 & 1764 & 81 & 378 \\
\hline 16 & 23 & 4 & 529 & 16 & 92 \\
\hline 17 & 41 & 8 & 1681 & 64 & 328 \\
\hline 18 & 26 & 2 & 676 & 4 & 52 \\
\hline 19 & 31 & 3 & 961 & 9 & 93 \\
\hline 20 & 20 & 2 & 400 & 4 & 40 \\
\hline 21 & 33 & 6 & 1089 & 36 & 198 \\
\hline 22 & 37 & 2 & 1369 & 4 & 74 \\
\hline 23 & 23 & 2 & 529 & 4 & 46 \\
\hline 24 & 40 & 6 & 1600 & 36 & 240 \\
\hline 25 & 28 & 5 & 784 & 25 & 140 \\
\hline 26 & 32 & 6 & 1024 & 36 & 192 \\
\hline 27 & 16 & 0 & 256 & 0 & 0 \\
\hline 28 & 33 & 5 & 1029 & 25 & 165 \\
\hline 29 & 32 & 5 & 1024 & 25 & 160 \\
\hline \multirow[t]{2}{*}{30} & 29 & 0 & 841 & 0 & 0 \\
\hline & 901 & 120 & 28389 & 670 & 3977 \\
\hline
\end{tabular}

$\mathrm{p}<0.01$ 
Cuadro 3: Puntajes en la PCBB (X) y en la PP de Matemática ( $\mathrm{Y}$ )

\begin{tabular}{|c|c|c|c|c|c|}
\hline $\mathrm{n}$ & $\mathrm{X}$ & $Y$ & $\mathrm{Xi}^{2}$ & $Y i^{2}$ & $\overline{X i Y i}$ \\
\hline 1 & 32 & 7 & 1024 & 49 & 224 \\
\hline 2 & 33 & 8 & 1089 & 64 & 264 \\
\hline 3 & 38 & 7 & 1444 & 49 & 266 \\
\hline 4 & 36 & 5 & 1296 & 25 & 190 \\
\hline 5 & 38 & 7 & 1444 & 49 & 252 \\
\hline 6 & 26 & 3 & 676 & 9 & 78 \\
\hline 7 & 25 & 5 & 625 & 25 & 125 \\
\hline 8 & 22 & 2 & 484 & 4 & 44 \\
\hline 9 & 31 & 9 & 961 & 81 & 279 \\
\hline 10 & 30 & .7 & 900 & 49 & 210 \\
\hline 11 & 29 & 2 & 841 & 4 & 58 \\
\hline 12 & 33 & 5 & 529 & 25 & 115 \\
\hline 13 & 28 & 8 & 784 & 64 & 224 \\
\hline 14 & 26 & 3 & 676 & 9 & 78 \\
\hline 15 & 42 & 9 & 1764 & 81 & 378 \\
\hline 16 & 23 & 8 & 529 & 64 & 184 \\
\hline 17 & 41 & 10 & 1681 & 100 & 410 \\
\hline 18 & 26 & 3 & 676 & 9 & 78 \\
\hline 19 & 31 & 8 & 961 & 64 & 248 \\
\hline 20 & 20 & 6 & 400 & 36 & 120 \\
\hline 21 & 33 & 7 & 1089 & 49 & 231 \\
\hline 22 & 37 & 8 & 1369 & 64 & 296 \\
\hline 23 & 23 & 2 & 529 & 4 & 46 \\
\hline 24 & 40 & 8 & 1600 & 64 & 320 \\
\hline 25 & 28 & 8 & 784 & 64 & 320 \\
\hline 26 & 32 & 8 & 1024 & 64 & 256 \\
\hline 27 & 16 & 0 & 256 & 0 & 0 \\
\hline 28 & 33 & 6 & 1029 & 36 & 198 \\
\hline 29 & 32 & 7 & 1024 & 49 & 224 \\
\hline \multirow[t]{2}{*}{30} & 29 & 5 & 841 & 25 & 145 \\
\hline & 901 & 181 & 28389 & 1279 & 5765 \\
\hline
\end{tabular}

$p<0.01$ 


\section{Discusión}

La finalidad de este estudio fue explorar y resolver en alguna medida la interrogante acerca de la relación entre el conocimiento de conceptos básicos y el rendimiento escolar. Para ello nos planteamos la pregunta ¿existe relación significativa entre los puntajes de la prueba PCBB y los puntajes de la PP?

De acuerdo al análisis estadístico aplicado a los datos, podemos decir que los resultados muestran una buena correlación entre las variables de estudio y además que a mayores puntajes en la prueba de conceptos mayor rendimiento escolar. Sin embargo, hacemos esta inferencia con la limitación que caracteriza a la muestra, es decir que sólo podemos proyectar estos resultados a la población en la cual se realizó el estudio.

Sería recomendable por tanto ensayar un estudio en el cual se tome una muestra probabilística y se verifique si se mantiene la correlación entre las variables, incluso en los diferentes estratos socioeconómicos.

El promedio obtenido por este grupo, en la prueba de conceptos, es similar al encontrado por Narváez (1987) en el nivel socioeconómico bajo, puntuaciones que son consideradas bajas en relación con las del nivel socioeconómico medio y alto de su estudio.

Este bajo rendimiento en la prueba de conceptos nos estaría señalando una deficiencia en la adquisición de los mismos y por lo tanto, estas niñas llegan al primer grado sin tener los conceptos básicos requeridos para acceder con éxito a las demandas del programa educativo. Este hallazgo puede considerarse importante ya que según Boehm, la prueba permite la detección e identificación temprana, no sólo de las nociones que necesitarán ser reforzadas sino también de los niños que requerirán de un programa de instrucción que compense sus carencias.

El problema es que la adquisición de estos conceptos básicos, en la mayoría de los casos se da al interior del núcleo familiar y durante los primeros años de vida. En relación a esto se presentan dos cuestiones importantes. La primera es que como consecuencia lógica de lo anterior, dichos conceptos no sólo no forman parte de los objetivos instruccionales, en el primer grado, sino que generalmente, el maestro los incorpora como 
parte de las consignas que utiliza, lo cual dificulta aún más la comprensión, así como la adecuada ejecución de la tarea, colocándolo en una situación de desventaja para realizar un aprendizaje eficaz.

La segunda cuestión se relaciona con lo referido por Bernstein (1967), cuando señala que los niños pertenecientes a la clase socioeconómica baja se desarrollan en un medio, en el cuàl se utilizan "códigos restringidos" para la comunicación y que por ende, las carencias que esto genera repercutirán en los aprendizajes futuros. Sin embargo, estas condiciones no son irreversibles, de tal forma que, si el niño recibiera una mayor estimulación en el área del lenguaje, se incrementaría el desarrollo de su vocabulario y en consecuencia se facilitaría la adquisición de conceptos (Ausubel, 1981).

Relacionado con lo anterior, se puede apreciar la repercusión que tiene en los niños la privación sociocultural, que en muchos casos, va acompańada de episodios se desnutrición en la primera infancia, situación que traerá consigo un retraso en todas las áreas de desarrollo (Pollitt, 1974). Hallazgos similares a estos han sido obtenidos por Majluf (1988) en relación a los efectos negativos que la desnutrición crónica produce en el aprendizaje de la lectura y las matemáticas, mientras que la carencia de estimulación sociocultural afecta principalmente, el rendimiento intelectual. Por su parte, Reátegui et al. (1975) al estudiar la influencia de la variable socioeconómica en la formación de estructuras cognitivo-afectivas, encontraron que los niños de zonas marginales mostraban mayores dificultades en cuanto a pensamiento abstracto, simbolización y conceptualización.

Otro factor a considerar en la realización de un estudio similar sería que la aplicación de la prueba pedagógica se efectúe en forma individual y no grupal, para así poder tener un mayor control de aquellas variables que puedan afectar el desempeño de niño en la prueba. En el caso de la presente investigación, se ha observado, la tendencia que tienen los niños a copiarse y a estar pendientes del rendimiento de sus compañeros.

Dado que la $\mathrm{PCBB}$ nos permite una detección temprana, en cuanto a conceptos básicos, se recomienda utilizarla, en nuestro medio con la finalidad de identificar el nivel de conocimiento que sobre el particular tienen los niños al ingresar al Centro Educativo. De esta manera se podría 110 
reforzar las áreas en las que se encuentren deficiencias. Si el maestro pudiese tener la posibilidad de contar con esta información, al inicio del año escolar podría efectuar una mejor planificación de la enseñanza, apuntando a evitar el fracaso escolar o la repitencia, que tienen una alta incidencia sobre todo en el primer grado.

\section{Referencias}

Arnau, J. (1986). Psicología Experimental. México: Editorial Trillas Ausubel, D.P. (1976). Psicología Educativa. Un punto de vista cognoscitivo. México: Editorial Trillas.

Bernstein, (1967). Social Structure, language and learning. En J.P. de Cecco (Ed.). The Psychology of language, thought and instruction. Nueva York: Holt Rinehart and Winston.

Boehm, A. (1971). Boehm Test of Basic Concepts. Nueva York: The Psychological Corporation.

Boehm, A. (1980). Test Boehm de Conceptos Básicos. Madrid: TEA.

Boehm, A. (1986). Boehm Test of Basic Concepts (Revised). Nueva York: The Psychological Corporation.

Bourne, L. y Restle, (1975). Psicología del Pensamiento. México: Editorial Trillas.

Ellis, H.C. (1980). Fundamentos del Aprendizaje y procesos cognoscitivos del hombre. México: Editorial Trillas.

Majluf, A. (1988). Relación entre el estado nutricional y la estimulación ambiental con el rendimiento cognitivo y escolar del niño del primer grado de la zona central media del Perú. Revista de Psicología de la PUCP. 6, 1-2, 5-17.

Marín, G. (1986). Consideraciones metodológicas básicas para conducir investigaciones psicológicas en América Latina. Acta Psiquiátrica y Psicológica en América Latina, 32, 184.

Mussen, P. Conger, J. y Kagan, J. (1985). Desarrollo de la personalidad en el niño. México: Editorial Trillas.

Narváez, A. (1987). Prueba de Conceptos Básicos de Boebm: Estudio comparativo en niños de diferente nivel socioeconómico de Lima y Callao. Tesis de Bachiller. PUCP.

Oerter, R. (1964). Psicología del Pensamiento, Barcelona: Herder. 
Pollitt, E. (1982). Desnutrición, Inteligencia y Politica Social. Lima: Studium. Reátegui, N. y colbs. (1975). Estudio de la formación de estructuras cognitivoafectivas básicas en niños sujetos a nuevos programas de enseñanza. Lima: INIDE.

Thorne, C. y Narváez, A. (1987). La prueba de Conceptos Básicos de Boehm: adaptación y elaboración de baremos para Lima y Callao. Revista de Psicología de la PUCP, 5, 2, 135-148.

Vigotsky, L. (1992). Pensamiento y Lenguaje. Buenos Aires: Ediciones Fausto. 